Supporting Information for

\title{
A SPR-based membrane protein-targeted active ingredients recognition strategy: construction and implementation in ligand screening from herbal medicines
}

Langdong Chen ${ }^{1, \dagger}$, Diya Lv ${ }^{1, \dagger}$, Shaozhan Wang ${ }^{\ddagger}$, Dongyao Wang ${ }^{\dagger}$, Xiaofei Chen ${ }^{1, \dagger}, Y_{u e}$ Liu $^{\dagger}$, Zhanying Hong ${ }^{\dagger}$, Zhenyu Zhu ${ }^{\dagger}$, Yan $\mathrm{Cao}^{*}, \dagger$, Yifeng Chai ${ }^{*}$,

$\uparrow$ School of Pharmacy, Second Military Medical University, Shanghai 200433, PR China

† Department of Pharmacy, Shanghai 9th People's Hospital, Shanghai Jiao Tong University School of Medicine, Shanghai 201999, PR China

TABLE OF CONTENT

A. Supporting Experimental Section

- Materials and reagents

- Cell culture

- Lentiviral particle production

- LVPs purification.

- LVP-beads staining.

- LVPs immobilization condition scouting

- Recovery of CXCR4-bound ingredients

- Experimental condition for UPLC-QTOF-MS analysis

- Suitability determination

- Robustness determination

- Preparation of sample extracts.

- Ligand screening

- Molecular docking

B. Supporting Figures and Tables

- Fluorescence intensity of $293 \mathrm{~T}$ cells

- LVPs immobilization condition scouting

- Suitability determination

- Robustness determination

- Ligand screening

- Molecular docking 


\section{Supporting Experimental Section}

Materials and reagents. Lentiviral particle packaging plasmids were gifts from Didier Trono, including pLVTHM, psPAX2, and pMD2G1 . CXCR4 interference shRNA plasmid was designed and synthetized (pGPU6/GFP/Neo-shRNA vector, 5'CCAUGAAGGAACCCUGUUUTT-3'; Genepharma, Shanghai, China). Antibodies were purchased from Abcam, including CXCR4, p24 and GAPDH. Standard compounds (AMD 3100, gefitinib, stattic, purity > 99.9\%) were purchased from Meilunbio (Dalian, China). Methanol, acetonitrile and formic acid (Sigma Aldrich, Missouri, United States) were all HPLC-grade. Materials and reagents used for SPR assays (including CM5 chips, EDC, NHS and ethanolamine) were purchased from GE Healthcare (Shanghai, China). Medicinal herbs were purchased from Leiyunshang Pharmacy (Shanghai, China). All these medicinal herbs were authenticated by Lianna Sun (Department of Pharmacognosy, School of Pharmacy, Second Military Medical University, Shanghai, China). 8 - $\mu \mathrm{m}$-pore, $6.5-\mathrm{mm}$ polycarbonate transwell filters were purchased from Corning Costar (Cambridge, Massachusetts, United States).

Cell culture. HEK293T cells were purchased from Cell Bank of Shanghai Branch of Chinese Academy of Sciences. Cells were maintained in Dulbecco's minimum essential medium (DMEM) supplemented with 10\% fetal bovine serum (FBS), $100 \mathrm{U} / \mathrm{mL}$ penicillin, and $100 \mu \mathrm{g} / \mathrm{mL}$ streptomycin. The cells were cultured in a humidified atmosphere of $5 \% \mathrm{CO} 2$ at $37^{\circ} \mathrm{C}$.

Lentiviral particle production. LVPs were produced by JetPEI (Polyplus, Illkirch, France) cotransfection of HEK293T cells with pLVTHM, psPAX2 and pMD2G plasmids at a 1:1:1 ratio $^{2}$. 293T cells were divided into normal group and interference group. $10 \mu \mathrm{g}$ pGPU6/GFP/Neo-shRNA vector was transfected into cells in control group with JetPEI (shRNA: JetPEI=3:1). While $10 \mu \mathrm{g}$ shRNA plasmid was transfected into the cells in treated group with JetPEI. Three plasmids (pLVTHM, psPAX2 and pMD2G) were transfected into the two groups of $293 \mathrm{~T}$ cells $24 \mathrm{~h}$ after shRNA plasmid transfection. The fluorescence intensity was detected under a fluorescence microscope after transfection of 24, 48 and $72 \mathrm{~h}$. 
LVPs purification. Polyethylene glycol 8000 (PEG8000) was used to purify LVPs. Supernatant containing LVPs was mixed with PEG8000 at 4:1 ratio. The solution was mixed gently for 5 times every 30 mins. The solution was stored at $4{ }^{\circ} \mathrm{C}$ overnight and then centrifuged at $8000 \mathrm{~g}$ for $30 \mathrm{mins}$. The supernatant was removed and the sediment was resuspended with PBS. LVPs suspension was centrifuged at $8000 \mathrm{~g}$ for 30 mins. The supernatant was removed and the sediment was resuspended with PBS. Finally, LVPs were aliquoted and stored at $-80{ }^{\circ} \mathrm{C}$.

LVP-beads staining. CXCR4 on the surface of LVPs bound to latex beads was stained with antibodies by standard techniques ${ }^{3}$, details about the LVP-beads staining could be found in Supplementary Information. p24 was also labeled with antibodies for reference. CXCR4-APC antibody was added into the LVP-beads solution and incubated for $2 \mathrm{~h}$ at $4{ }^{\circ} \mathrm{C}$. Then the sample was centrifuged $\left(1950 \times \mathrm{g}, 5 \mathrm{~min}\right.$, ) at $4{ }^{\circ} \mathrm{C}$ and $1 \mathrm{~mL}$ PBS- $0.5 \%$ BSA was added for suspension. This procedure was replicated for three times. After wash, $300 \mathrm{~mL}$ PBS- $0.5 \%$ BSA was added for sample resuspension. Then the samples were injected into flow cytometry to detect the fluorescent intensity.

LVPs immobilization condition scouting. SPR assays were performed on Biacore T200 system (GE Healthcare, Sweden). The physical absorption response signal of LVPs on CM5 chips with four different $\mathrm{pH}$ buffers $(4.0,4.5,5.0$, and 5.5) was tested to find the optimal buffer. Then the physical absorption response signal of LVPs on CM5 chips at four different concentrations $(200,300,400$ and $500 \mu \mathrm{g} / \mathrm{mL}$, use total protein concentration of LVPs as reference) was detected to find the optimal concentration of LVPs. Then LVPs were diluted in $10 \mathrm{mM}$ sodium acetate at the optimal condition $(\mathrm{pH}=4.0,500 \mu \mathrm{g} / \mathrm{mL})$ and then immobilized on detection cells by applying EDC/NHS cross-linking reaction. For affinity detection module, flow cell (FC) 1 was immobilized with LVPs with low CXCR4 expression (CXCR4-LVPs) and FC2 was immobilized with LVPs with high CXCR4 expression (CXCR4+-LVPs). For ligand screening module, all the four FCs on activity chip were immobilized with 
CXCR4 ${ }^{+}$-LVPs and all the four FCs on reference chip were immobilized with CXCR4--LVPs.

Recovery of CXCR4-bound ingredients. Herbal extract was injected over the sensor surface for $180 \mathrm{~s}$ at $5 \mu \mathrm{L} / \mathrm{min}$. Phosphate buffer saline (PBS) with 5\% DMSO was selected as running buffer. The flow system was washed with $0.5 \%$ formic acid. A small volume of $2 \mu \mathrm{L}$ sample was injected into the flow cells and incubated for 20 s. Then, the flow direction over the sensor surface was reversed and the sample containing CXCR4-bound ingredients was deposited in $10 \mu \mathrm{L}$ ammonium bicarbonate $(50 \mathrm{mM})$. In a cycle, the recovery procedure was replicated for 5 times.

Experimental condition for UPLC-QTOF-MS analysis. Herbal extracts and SPR-recovered samples were analyzed on an Agilent 1290 series UPLC system (Agilent Corp., USA). Chromatographic separation was carried out on a Waters XSelect ${ }^{\circledR}$ HSS T3 $2.5 \mu \mathrm{M}$ column $(2.1 \times 100 \mathrm{~mm})$ at $25^{\circ} \mathrm{C}$. The injection volume is 5 $\mu \mathrm{L}$. The mobile phase consisted of $0.1 \%$ aqueous formic acid (v/v) (A) and acetonitrile (B), using a gradient elution. Elution procedure is $10 \% \mathrm{~B}$ at $0-1 \mathrm{~min}, 10-$ $90 \% \mathrm{~B}$ at 1-7 $\mathrm{min}$, and $90 \% \mathrm{~B}$ at 7-15 min for SPR-recovered samples. The flow rate was kept at $3.5 \mathrm{~mL} / \mathrm{min}$, and a post-column split was used to maintain a flow rate of $0.4 \mathrm{~mL} / \mathrm{min}$ into the mass spectrometer source to obtain good nebulization efficiency. Detection was performed by an Agilent 6538 UHD Accurate-Mass QTOF/MS (Agilent Corp., USA). The analysis was performed using full-scan mode and mass range was set at m/z 100-1000 in positive ion mode. The conditions of ESI source were as follows: drying gas $(\mathrm{N} 2)$ flow rate, $10 \mathrm{~L} / \mathrm{min}$; drying gas temperature, $350{ }^{\circ} \mathrm{C}$; nebulizer, 35 psig; capillary voltage, $4000 \mathrm{~V}$; fragmentor voltage, $120 \mathrm{~V}$; skimmer voltage, $60 \mathrm{~V}$; octopole RF, $250 \mathrm{~V}$. All the data were processed by Agilent MassHunter Software ver. B.02.00 (Agilent Technologies). Tuning mix (G196985000, Agilent Corp., USA) was used for lock mass calibration in the assay.

Robustness determination of ligand screening module. Subsequently, the robustness of the LVP-SPR system was validated by constructing a complex system. AMD3100 solution $(20 \mu \mathrm{M})$, gefitinib solution $(20 \mu \mathrm{M})$ and the extract solution of Bark 
Cinnamon (R. Corydalis, $100 \mathrm{ng} / \mathrm{mL}$ ) were mixed in PBS. The samples were centrifuged at $5000 \mathrm{~g}$ for 3 mins and then injected into SPR for compound capture. Recovery experiment was performed for 50 cycles. Recovered samples were dried by nitrogen gas and dissolved in $100 \mu \mathrm{L}$ methanol. The samples were centrifuged at 5000 $\mathrm{g}$ for 3 mins and the supernatant was injected into UPLC-QTOF-MS system for analysis.

Preparation of sample extracts. The crude drugs were smashed into powders and then sieved through a 40-mesh sieve. Powder samples in $1 \mathrm{~g}$ were extracted by supersonic extraction with $10 \mathrm{~mL}$ ethanol: $\mathrm{H}_{2} \mathrm{O}$ (80: 20) for 30 mins. Subsequently, the extracts were centrifuged and filtered through a $0.22-\mu \mathrm{m}$ filter. The samples were stored at room temperature.

Ligands screening. Herbal extracts were recovered for 10 cycles. Recovered samples were dried by nitrogen gas and dissolved in methanol. Recovery samples were centrifuged at $5000 \mathrm{~g}$ for 3 mins and then injected into UPLC-QTOF-MS system for analysis. Herbal extracts were diluted in methanol and then injected into UPLCQTOF-MS. Chemical information about constituents in herbal medicines were collected from Traditional Chinese Medicine integrative database (TCMID) ${ }^{4}$. According to the parameters provided by the Agilent Qualitative Analysis software, such as mass accuracy, isotope abundance match, spacing match and double bond equivalent index, components were confirmed due to the fine matching of these parameters.

Molecular docking. The crystal structure of CXCR4 was downloaded from PDB database $(\mathrm{PDB} I D=3 \mathrm{ODU})^{5}$. Water molecules were removed from the structure. Chemical structure of senkyunolide I was downloaded from PubChem database and optimized in Discovery Studio 3.0. Chemical structures were all saved as mol 2 format. The docking site was defined at the center of the position of original ligand with $10 \AA$ radius. Molecular docking was performed by employing the CDOCKER module in the DS 3.0 package. Top hits was set as 100 and poster cluster radius was set as 0.5 . The other options were set as default settings. CDOCKER 
energy was calculated to evaluate the best pose. The docking results were visualized in pymol. 


\section{Supporting Figures and Tables}

Fluorescence intensity of $293 \mathrm{~T}$ cells. In order to produce lentiviral particles, 293T cells were divided into two groups, vector was transfected into cells from the control group, while shRNA plasmid was transfected into the $293 \mathrm{~T}$ cells from treated group to inhibit the expression of CXCR4. Cells in control group produce CXCR4+-LVP and cells in treated group produce CXCR4--LVP. Three LVP-packaging plasmids were transfected into the two groups of $293 \mathrm{~T}$ cells $24 \mathrm{~h}$ after shRNA transfection. The fluorescence intensity was detected under a fluorescence microscope after transfection of 24,48 and $72 \mathrm{~h}$. It was shown that the fluorescence intensity increased as time went on (Figure S1), which demonstrated that the LVPs were successfully produced in two groups of $293 \mathrm{~T}$ cells.

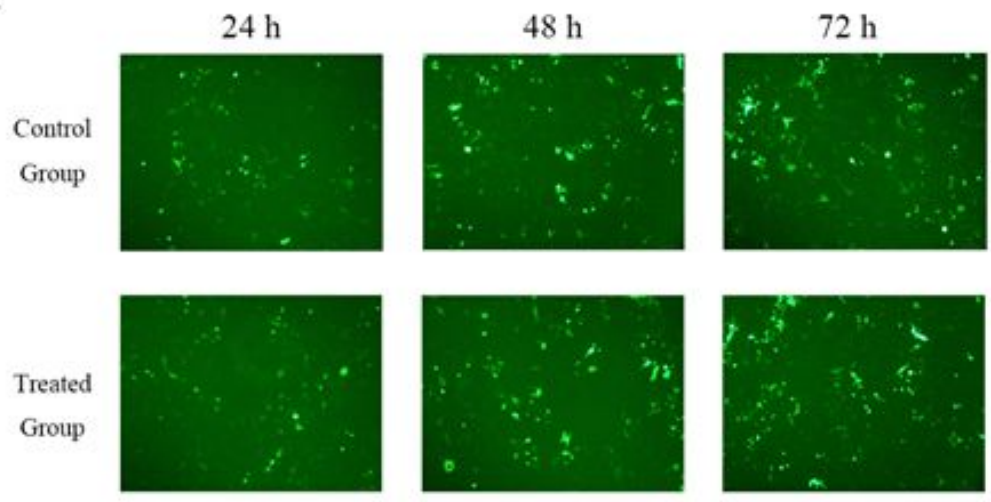

Figure S1. The fluorescence intensity of 293T cells after transfection of shRNA and three plasmids.

LVPs immobilization condition scouting. In order to immobilize LVPs on SPR chips with good activity and high response, we scouted the optimal condition for LVP immobilization. First, we investigated the influence of buffer $\mathrm{pH}$ on LVPs physically absorbed to CM5 chips. We tested the physical absorption response signal of LVPs on CM5 chips with four different $\mathrm{pH}$ buffers $(4.0,4.5,5.0$, and 5.5). The results were shown in Figure S2A. It was obvious that LVPs in $\mathrm{pH} 4.0$ buffer could bind to CM5 with the highest response. Then we chose $\mathrm{pH} 4.0$ buffer and tested the physical absorption response signal of LVPs on CM5 chips at four different concentrations (200, 300,400 and $500 \mu \mathrm{g} / \mathrm{mL}$, use total protein concentration of LVPs as reference). The results were shown in Figure S2B. It revealed that LVPs at $500 \mu \mathrm{g} / \mathrm{mL}$ could bind to 
CM5 with the highest response. Finally, $\mathrm{pH} 4.0$ buffer and $500 \mu \mathrm{g} / \mathrm{mL}$ LVPs concentration were determined as the optimal immobilization conditions.
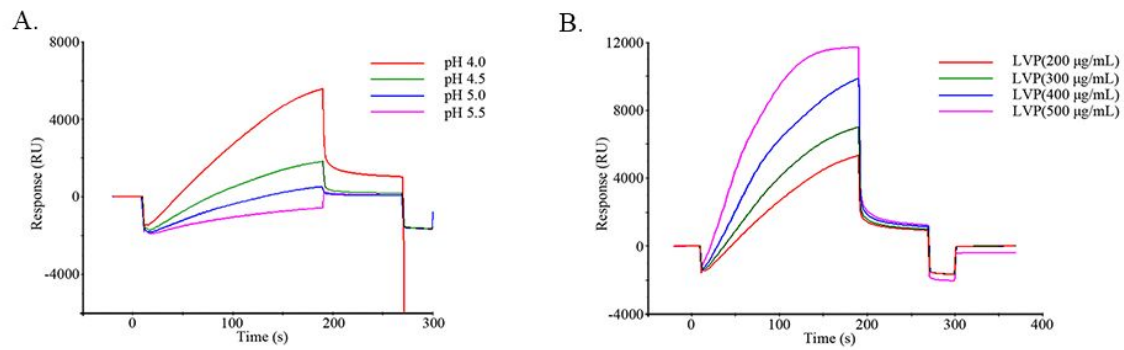

Figure S2. Immobilization conditions scouting, including buffer $\mathrm{pH}(\mathrm{A})$ and LVPs concentration (B)

Suitability determination. The suitability of the ligand screening module was investigated. Three typical compounds, AMD3100, gefitinib and stattic, were recovered on the activity chip and reference chip respectively. The recovered samples and standard compounds were analyzed by UPLC-QTOF-MS system. The chromatographic analysis results of three compounds were shown in Table $\mathrm{S} 1$. The $\mathrm{R}_{t}$ of AMD3100 standard was $10.268 \mathrm{~min}$ and the $\mathrm{m} / z$ of the $([\mathrm{M}+\mathrm{H}]+)$ ion was 503.4541 . The recovery amounts of AMD3100 on the activity chip and the reference chip were compared. There is a significant difference between the recovery amounts on the two chips. This result suggests that CXCR4 specific ligands could be recognized by the system.

The $\mathrm{R}_{\mathrm{t}}$ of gefitinib standard was $5.063 \mathrm{~min}$ and the $m / z$ of the $([\mathrm{M}+\mathrm{H}]+)$ ion was 447.1596. The recovery amounts of gefitinib on activity chip and the reference chip were also compared. There is no significant difference between the recovery amounts on the two chips. The $\mathrm{R}_{\mathrm{t}}$ of stattic standard was $7.265 \mathrm{~min}$ and the $\mathrm{m} / z$ of the $([\mathrm{M}+\mathrm{H}]+)$ ion was 212.0012. While stattic could not be recovered and identified by the system.

Table $\mathbf{S 1} \mathrm{R}_{\mathrm{t}}, \mathrm{m} / \mathrm{z}$ and peak area of AMD3100, gefitinib and stattic standards and recovery samples on two chips

\begin{tabular}{ccccccccccc}
\hline & \multicolumn{3}{c}{ Standard compound } & \multicolumn{3}{c}{ Recovery sample on activity } & \multicolumn{3}{c}{ Recovery sample on reference } \\
& \multicolumn{3}{c}{} & \multicolumn{3}{c}{ chip } & \multicolumn{3}{c}{ chip } \\
& $\mathrm{R}_{\mathrm{t}}$ & $\mathrm{m} / \mathrm{z}$ & Peak area & $\mathrm{R}_{\mathrm{t}}$ & $\mathrm{m} / z$ & Peak area & $\mathrm{R}_{\mathrm{t}}$ & $\mathrm{m} / z$ & Peak area \\
\cline { 2 - 11 } AMD3100 & 10.268 & 503.4541 & 8026434.48 & 10.270 & 503.4575 & 281555.29 & 10.288 & 503.4541 & 141739.05 \\
Gefitinib & 5.063 & 447.1596 & 67255703.99 & 5.134 & 447.1596 & 175840.20 & 5.126 & 447.1619 & 164767.83 \\
Stattic & 7.265 & 212.0012 & 734655.45 & - & - & - & - & - & - \\
\hline
\end{tabular}


Robustness determination. The peak area of AMD3100 on activity chip was 169148.2

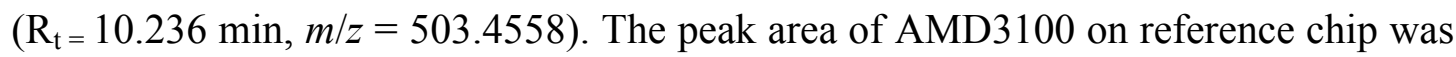
$78850.27\left(\mathrm{R}_{\mathrm{t}}=10.286 \mathrm{~min}, \mathrm{~m} / z=503.4542\right)$. It was found that the recovery amount of AMD3100 on the activity chip has significant difference compared with the recovery amount on the reference chip. In addition, the peak area of gefitinib on activity chip was $23557\left(\mathrm{R}_{\mathrm{t}}=5.088 \mathrm{~min}, m / z=447.1554\right)$. The peak area of gefitinib on activity chip was $23557\left(\mathrm{R}_{\mathrm{t}}=5.087 \mathrm{~min}, \mathrm{~m} / \mathrm{z}=447.1612\right)$. It is shown that the recovery amount of gefitinib has no significance on the two chips (Table S2).

Table S2 $\mathrm{R}_{\mathrm{t}}, m / z$ and peak area of AMD3100 and gefitinib on two chips

\begin{tabular}{ccccccc}
\hline & \multicolumn{3}{c}{ Activity chip } & \multicolumn{3}{c}{ Reference chip } \\
\cline { 2 - 7 } & $\mathrm{R}_{\mathrm{t}}$ & $m / z$ & Peak area & $\mathrm{R}_{\mathrm{T}}$ & $m / z$ & Peak area \\
\cline { 2 - 7 } AMD3100 & 10.236 & 503.4558 & 169148.2 & 10.286 & 503.4542 & 78850.27 \\
Gefitinib & 5.088 & 447.1554 & 23557 & 5.087 & 447.1612 & 24838.7 \\
\hline
\end{tabular}

Ligands screening. In order to eliminate the false positive result, we confirmed the screening result according to the peak area of gefitinib in both recovery samples (Figure $\mathrm{S} 3$ ). In recovery sample from activity chip, the $R_{t}$ of gefitinib was $5.056 \mathrm{~min}$. The $\mathrm{m} / \mathrm{z}$ of parent ion was 447.1589 and the area of chromatographic peak was 14660.23 . In recovery sample from reference chip, the $R_{t}$ of gefitinib was $5.033 \mathrm{~min}$. The $\mathrm{m} / \mathrm{z}$ of parent ion was 447.1592 and the area of chromatographic peak was 14367.68. Analysis results of senkyunolide I and gefitinib in two recovery samples were shown in Table S3. It is shown that the recovery of senkyunolide I has significant difference between activity chip and reference chip, while the recovery of gefitinib has no significance on the two chips. Hence, senkyunolide I was identified as a candidate compound from Chuanxiong extract sample by using ligand screening module. 

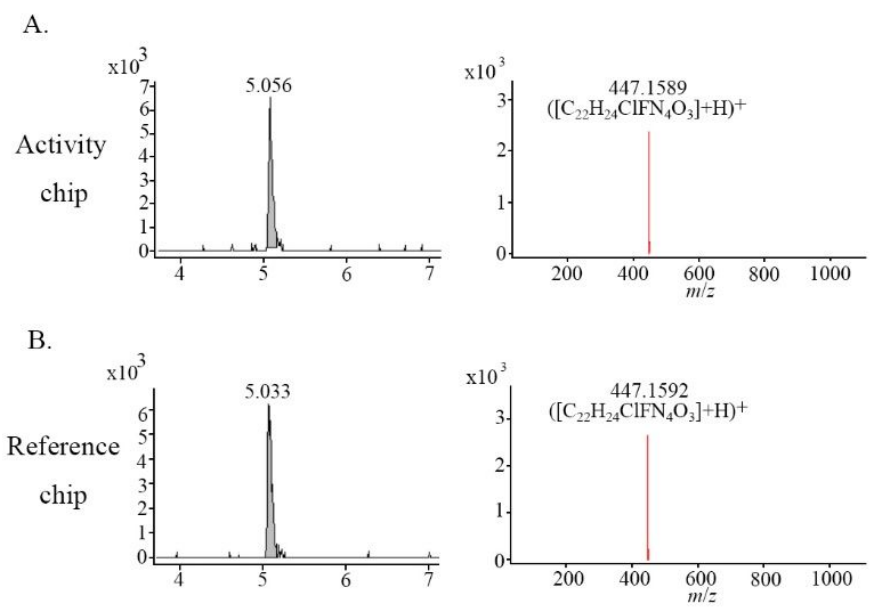

Figure S3. There is no significant difference between the recovery amounts of gefitinib mix in herbal extract on activity chip (A) and reference chip (B).

Table S3 $\mathrm{R}_{\mathrm{t}}, m / z$ and peak area of senkyunolide I and gefitinib on two chips

\begin{tabular}{ccccccc}
\hline & \multicolumn{3}{c}{ Activity chip } & \multicolumn{3}{c}{ Reference chip } \\
\cline { 2 - 7 } & $\mathrm{R}_{\mathrm{t}}$ & $m / z$ & Peak area & $\mathrm{R}_{\mathrm{t}}$ & $m / z$ & Peak area \\
\cline { 2 - 7 } Senkyunolide I & 6.442 & 247.0944 & 63405.87 & 6.447 & 247.0941 & 28418.47 \\
Gefitinib & 5.056 & 447.1589 & 14660.23 & 5.033 & 447.1592 & 14367.68 \\
\hline
\end{tabular}

Molecular docking. Molecular docking was performed. Senkyunolide I and AMD3100 were docking in the active site of CXCR4 by Discovery Studio 3.0. The molecular docking results indicated that senkyunolide I could effectively bind to the active site of CXCR4 (Figure S4), Cys-186 amino acid residue could form hydrogen bond with hydrogen atom of 6- hydroxyl group on isobenzofuranone ring, His-113 amino acid residues could form hydrogen bond with oxygen atoms of 6- and 7-hydroxyl group on isobenzofuranone ring, Trp-94 could form Pi-Pi interaction with on isobenzofuranone ring, Leu-41 and Ala-98 amino acid residues could form alkyl-alkyl interaction with butylidene. The CDOCKER ENERGY was $-20.4699 \mathrm{~kJ} / \mathrm{mol}$. The results of SPR assay and molecular docking revealed that senkyunolide I could effectively bind to CXCR4 and could be a potential CXCR4 antagonist. 


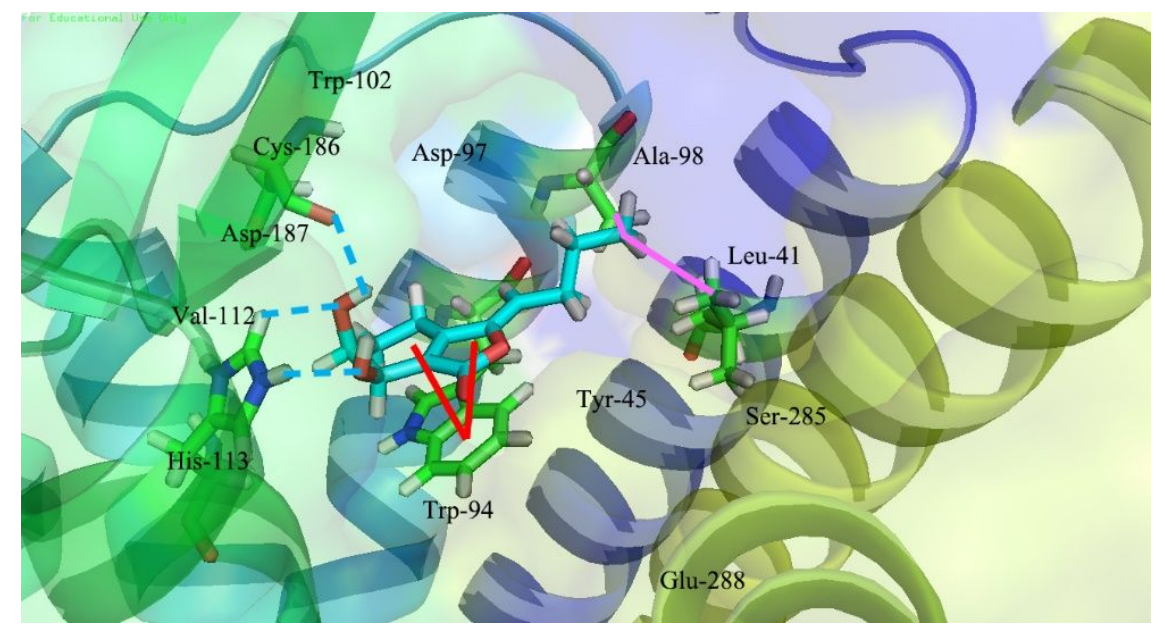

Figure S4. The binding mode between CXCR4 and senkyunolide I predicted by CDOCKER.

\section{References}

(1) Wiznerowicz, M.; Trono, D. Conditional suppression of cellular genes: lentivirus vector-mediated drug-inducible RNA interference [J]. Journal of virology 2003, 77, 8957-8961.

(2) Martinez-Munoz, L.; Barroso, R.; Paredes, A. G.; Mellado, M.; Rodriguez-Frade, J. M. Methods to immobilize GPCR on the surface of SPR sensors [J]. Methods in molecular biology 2015, 1272, 173188.

(3) Frade, J. M.; Mellado, M.; del Real, G.; Gutierrez-Ramos, J. C.; Lind, P.; Martinez, A. C. Characterization of the CCR2 chemokine receptor: functional CCR2 receptor expression in B cells [J]. Journal of immunology (Baltimore, Md. : 1950) 1997, 159, 5576-5584.

(4) Huang, L.; Xie, D.; Yu, Y.; Liu, H.; Shi, Y.; Shi, T.; Wen, C. TCMID 2.0: a comprehensive resource for TCM [J]. Nucleic acids research 2018, 46, D1117-d1120.

(5) Wu, B.; Chien, E. Y.; Mol, C. D.; Fenalti, G.; Liu, W.; Katritch, V.; Abagyan, R.; Brooun, A.; Wells, P.; Bi, F. C.; Hamel, D. J.; Kuhn, P.; Handel, T. M.; Cherezov, V.; Stevens, R. C. Structures of the CXCR4 chemokine GPCR with small-molecule and cyclic peptide antagonists [J]. Science (New York, N.Y.) 2010, 330, 1066-1071. 